\title{
Desarrollo de la motivación en estudiantes de secundaria mediante la implementación de un programa educativo en Artes Plásticas basado en el Aprendizaje Cooperativo
}

\author{
Development of motivation in secondary students through the \\ implementation of an educational program in Plastic Arts based \\ on Cooperative Learning
}

\section{Implementação de um programa educacional de artes plásticas baseado no Aprendizagem Cooperativo sobre motivação em estudantes do colégio}

ISSN 1688-9304 - DOI: 10.18861/cied.2020.11.2.2990

Efraín Romero Artavia*1

https://orcid.org/0000-0002-7652-9133

Andrea Mora Campos ${ }^{* \star 2}$

https://orcid.org/0000-0001-9813-2674

Wendy González Víquez **3

https://orcid.org/0000-0003-2836-8096

Patricia Renee Garbanzo Hernández ${ }^{* * * 4}$

https://orcid.org/0000-0002-2027-6990

José Andrey Zamora-Araya****5

http://orcid.org/0000-0001-6050-5850

Fabiola Quirós ${ }^{* * * * * 6}$

https://orcid.org/0000-0003-2214-9044

Silvia García Vargas ${ }^{* * \star * * * 7}$

https://orcid.org/0000-0002-0960-3567_

Fecha de recibido: 22/08/2019

Fecha de aceptado: 13/03/2020

\section{Resumen}

Esta investigación analiza el desarrollo de la motivación de estudiantes de octavo año de una institución de educación pública de Costa Rica en la clase de Artes Plásticas (AP)i mediante la implementación de un programa educativo basado en el Aprendizaje Cooperativo (AC) Johnson, Johnson \& Holubec, 1994). En cuanto a instrumentos y materiales, para realizar la investigación se adaptó y validó la Escala de Motivación Académica (EMA) (Manassero y Vázquez, 2000) para determinar la Motivación (M) en estudiantes de enseñanza secundaria. Para el proceso de recolección de la información se utilizaron técnicas cualitativas tales como el cuestionario y la observación con el fin de evidenciar los diferentes aspectos de la motivación. La población participante estuvo conformada por estudiantes de octavo año, entre los 13 y 15 años de edad. Asimismo se emplearon dos grupos: uno de estudio y otro control. Se concluye que 
el desarrollo de la motivación mejoró en el grupo experimental con la intervención de las estrategias procedentes del AC desde el acercamiento cualitativo. No obstante, los métodos cuantitativos de recolección de datos no aportaron evidencia estadísticamente significativa a favor del grupo de estudio ya que el instrumento no fue sensible al paso del tiempo. Por tanto, esta escala no se recomienda para estudios de diseño longitudinal experimental.

Palabras clave: aprendizaje cooperativo, motivación, artes plásticas, secundaria, EMA.

\section{Abstract}

This research analyzes the development of motivation among eight-grade students at a public secondary school in Costa Rica in Plastic Arts classroom, through the implementation of an educational program based on Cooperative Learning (AC) Johnson, Johnson, \& Holubec, 1994). The Academic Motivation Scale (EMA) (Manassero \& Vázquez, 2000) was adapted and validated to determine the Motivation (M) among these students. Qualitative techniques such as questionnaires and observation were used to collect information, so as to make evident the different aspects of Motivation. The population under study consisted of eight-grade students, ranging from ages 13 to 15, divided in two groups: a study group and a control group. It was concluded that motivation development was improved in the experimental group with the intervention of cooperative learning strategies from a qualitative approach. However, quantitative methods for collecting information did not contribute with statistically significant evidence in favor of the study group, because the instrument was not sensitive to the passing of time. Therefore, this scale is not recommended for studies of experimental longitudinal design.

Keywords: cooperative learning, motivation, plastic arts, secondary education, AMS.

\section{Resumo}

Essa pesquisa analisa o desenvolvimento da motivação em alunos da oitava série de um colégio de educação pública da Costa Rica na aula de Artes Plásticas, através da implementação de um programa educacional baseado no Aprendizado Cooperativo (AC) Johnson, Johnson e Holubec, 1994). Quanto aos instrumentos e materiais para a realização da pesquisa, a Escala de Motivação Acadêmica (EMA) foi adaptada e validada (Manassero e Vásquez, 2000) para determinar a Motivação (M) em estudantes de ensino médio. No processo de coleta de informação, foram utilizadas técnicas qualitativas, como o questionário e a observação, aos fins de salientar os diferentes aspectos da M. A população participante esteve composta por estudantes da oitava série, com idades entre 13 e 15 anos, aliás, foram utilizados dois grupos, um de estudo e outro de controle. Conclusão: conclui-se que o desenvolvimento da motivação melhorou no grupo experimental com a intervenção das estratégias oriundas do AC de abordagem qualitativa, não obstante, as análises mostram que não há diferenças estatisticamente significativas no grupo de estudo, devido ao fato de que o instrumento não era sensível ao decorrer do tempo. Portanto, essa escala não é recomendada para análises experimentais de desenho longitudinal.

Palavras chave: aprendizagem cooperativa, motivação, artes visuais, colégio, ema. 


\section{Introducción}

Este estudio tuvo como objetivo analizar el desarrollo de la motivación en estudiantes de secundaria mediante la implementación de un programa educativo en AP basado en el AC.

Según Orozco (2013), Muñoz (2009) y Gilbert (2005) la falta de motivación es la causa del bajo desempeño académico del estudiantado, lo que constituye un problema cotidiano en clases de AP ya que esta materia usualmente es relegada con respecto a otras por no ser prioritaria (Romo, 1997). De tal forma es oportuno implementar estrategias que promuevan los intercambios sociales, la diversidad y el diálogo entre iguales como herramienta que permita intensificar el interés y la motivación en el aula.

Por lo antedicho, una metodología que puede colaborar en las clases de AP es la del Aprendizaje Cooperativo (AC). El mismo está constituido por una serie de estrategias e instrucciones de atención a la diversidad (Duran y Vidal, 2004) que según Duran y Monereo (2012) favorece al discente en su forma de aprender ya que se trabaja con grupos pequeños. Esto maximiza la asimilación de conocimientos de todos los integrantes del grupo al compartir experiencias, materiales y otras destrezas mediante el apoyo mutuo y con el establecimiento de metas comunes (Ferreiro y Espino, 2009) para lograr el aprendizaje propio y colectivo (Johnson \& Johnson, 1994). Todos los miembros del equipo son necesarios sin importar la labor que desempeñen porque cumplen una función dentro del grupo (Johnson, Johnson \& Holubec, 1994).

Según Duran y Vidal (2004) los grupos que trabajan con AC desarrollan una alta dependencia positiva entre los actuantes del grupo así como desenvolvimiento, resolución de problemas, pensamiento crítico, mejor autoestima y rendimiento académico (Ferreiro y Espino, 2009; Liao, 2006; Pujolàs, 2003). Otros aspectos importantes que fomenta esta metodología son el liderazgo, la alta interdependencia positiva, la interacción social que promueve un desarrollo elevado de pensamiento crítico y la adaptabilidad así como la madurez psicológica y emocional, de tal manera que produce mayores niveles de $\mathrm{M}$ en las clases (Ferreiro y Espino, 2009; Duran y Vidal, 2004; Pujolàs, 2003; Duran y Monereo, 2012; Lara 2001).

Investigaciones realizadas en el área de las APy AC en la enseñanza de música demostraron que el estudiantado mejoró aspectos tales como el autoconcepto académico respecto a un grupo control. Estos hallazgos también se han corroborado en otros ámbitos fuera del académico como, por ejemplo, el laboral (Ruiz, 2012).

El AC permite que los estudiantes entablen relaciones responsables y a largo plazo que los motivan a realizar sus tareas asignadas, cumplir con sus obligaciones y desarrollarse social y académicamente (Johnson, Johnson \& Smith, 1998) debido a que pueden llevar a cabo trabajos colectivos que fomentan la colaboración y el desarrollo escolar de unos a otros (Johnson, Johnson \& Holubec, 1994, p. 7).

Con base en lo anterior puede afirmarse que el AC fortalece la motivación. Tal como lo indican López (2009) y Panitz (1999) la motivación es uno de los efectos no cognitivos del AC, como una consecuencia de las situaciones afectivas, interpersonales y sociales resultantes de la cohesión grupal y que resulta eficaz para incrementar el rendimiento académico por la MI. Esta, a su vez, determina el rendimiento en expectativas futuras tales como persistencia, curiosidad, rendimiento y éxito. 
Dentro de las ventajas de esta metodología se encuentra la capacidad de generar espacios semejantes a la vida real tales como el trabajo en grupo (Chenich \& Santos, 2005), ya que cuando se incorpora el AC al quehacer diario normal las conductas de pedir y proporcionar ayuda se tornan genuinas y verdaderas, de tal manera que se mejora el desenvolvimiento social del estudiantado así como sus oportunidades de aprendizaje. Esto se produce porque el AC es parte de la naturaleza del ser humano, que es un ser social que convive en grupos de personas por diferentes motivos: supervivencia, procreación, diversión, motivos económicos u otros. De tal manera, al cooperar unos con otros se desarrollan facultades de convivencia que todo ser humano requiere para vivir en sociedad (Hauser, 1978).

Asimismo, en el AC los grupos estudian tanto el nivel de eficacia para lograr las metas propuestas como la medida de trabajo conjunto para garantizar una mejoría en su aprendizaje y, por ende, en su trabajo en equipo. Como resultado de este proceso los estudiantes logran conformarse en el mejor grupo posible en beneficio de ellos mismos (Johnson, Johnson \& Smith, 1998; Johnson, Johnson \& Holubec, 1994).

Por todo lo anterior el AC podría contribuir en las clases de AP (Johnson y Johnson, 1999) como aspecto favorable para el desarrollo de la M. Por dicha razón, con los insumos del estudiantado y la teoría del AC y AP, este estudio pretende implementar una propuesta innovadora en dichos estudiantes.

\section{Metodología}

\section{Participantes}

La institución seleccionada para implementar el estudio fue un liceo de educación pública de la provincia de Limón, Costa Rica. De un total de 13 grupos se seleccionaron dos de forma aleatoria y se denominaron grupo control y grupo experimental respectivamente. El primero trabajó con la metodología convencional. El segundo trabajó con la nueva metodología y se consideró con voz activa desde el inicio y durante la elaboración de la propuesta. Dicha propuesta pretende diseñar situaciones de AC que influyan positivamente en la M por parte del estudiantado. Ambos grupos se encuentran en octavo año y sus clases fueron impartidas por la misma profesora. Las edades de los participantes iban de 13 a 15 años (ver tabla 1).

Asimismo se organizaron tres grupos focales con el fin de complementar la información obtenida mediante las técnicas cuantitativas. En el primero de ellos, reunido al inicio de la investigación y en el que participó todo el estudiantado del grupo experimental, los discentes aportaron ideas de cómo querían recibir las lecciones de AP. Asimismo, para los otros dos grupos focales convocados al finalizar la investigación, del grupo experimental participaron siete estudiantes y ocho del grupo control escogidos a conveniencia, pues eran informantes clave, agregados a los dos desertores entrevistados.

\section{Procedimientos}

Se solicitaron los permisos pertinentes a la institución y a padres y madres de familia (se utilizaron compromisos de confidencialidad por la naturaleza mixta del estudio). Una vez obtenidas las autorizaciones se escogieron en forma aleatoria los grupos participantes. Con posterioridad se convocó al grupo focal inicial.

La investigadora principal, ocasionalmente, fue acompañada por otra, quien se encargó de realizar las observaciones de ambos grupos durante el procedimiento. 
Con posterioridad a este proceso se continuó con el diseño de un programa educativo en el cual se integraron los contenidos del programa de estudio del Ministerio de Educación Pública de Costa Rica (MEP), la teoría del AC y las sugerencias del estudiantado a partir del grupo focal inicial, con la colaboración de cinco especialistas en AP y AC.

Seguidamente se inició la aplicación de la escala EMA (Cokley, Bernard, Cunningham y Motoike, 2001) a ambos grupos. Específicamente, en este punto se realizó una comparación de los promedios en la escala EMA mediante una t-Student para descartar que los grupos tuvieran puntajes diferentes de motivación al inicio de la implementación y que esto pudiera afectar el estudio. Sin embargo, los promedios fueron estadísticamente diferentes (ver tabla 1). Una semana después volvió a aplicarse la EMA e inició la implementación del programa, que tuvo una duración de 12 semanas con dos lecciones semanales de 40 minutos. Consistió en cuatro unidades con una sola temática: La naturaleza como experimento, estilización y abstracción tomada del plan de estudios de octavo año del MEP. En todas las sesiones se realizaban observaciones de clase. Al finalizar la investigación se aplicó el instrumento EMA así como grupos focales para ambos grupos.

Tabla 1. Resultados de la prueba t-Student para comparar los grupos en el pretest.

\begin{tabular}{|c|c|c|c|c|}
\hline \multicolumn{5}{|c|}{ Comparación de grupos independientes } \\
\hline \multirow[t]{2}{*}{ Variable } & \multicolumn{3}{|c|}{ T-test de igualdad de medias } & \multirow{2}{*}{$\begin{array}{c}\begin{array}{c}\text { Test de Levene } \\
\text { para igualdad de } \\
\text { varianzas }\end{array} \\
\text { Sig. (2-colas) }\end{array}$} \\
\hline & $\mathrm{F}$ & Sig. & T & \\
\hline \multirow{2}{*}{ MIC } & 067 & ,797 & 2,166 & ,034 \\
\hline & & & 2,174 & 034 \\
\hline \multirow{2}{*}{ MIRRCO } & ,298 &, 587 & 2,528 & 014 \\
\hline & & & 2,510 & 015 \\
\hline \multirow{2}{*}{ MIPEE } & ,981 & ,326 & 3,130 &, 003 \\
\hline & & & 3,102 & ,003 \\
\hline \multirow{2}{*}{ MERE } & 1,978 & 165 & 4,030 & ,000 \\
\hline & & & 4,095 &, 000 \\
\hline \multirow{2}{*}{ MERI } & ,317 &, 575 & 2,346 & ,022 \\
\hline & & & 2,371 &, 021 \\
\hline \multirow{2}{*}{ MEI } & ,111 & ,740 & 4,032 &, 000 \\
\hline & & & 4,037 &, 000 \\
\hline \multirow{2}{*}{$\mathrm{ME}$} & ,000 & ,996 & 4,001 & ,000 \\
\hline & & & 4,023 & ,000 \\
\hline \multirow{2}{*}{ AMO } & 120 & ,730 & ,296 & ,769 \\
\hline & & & 294 & ,769 \\
\hline \multirow{2}{*}{ Total } & ,036 & ,850 & 3,773 & ,000 \\
\hline & & & 3,783 & ,000 \\
\hline
\end{tabular}

En la tabla anterior se observa que no hay diferencias estadísticamente significativas para los grupos $(p>, 05)$. Esto indica que los grupos no eran diferentes en los promedios al inicio de la investigación. 


\section{Instrumentos y técnicas utilizados}

\section{Escala de Motivación Académica (EMA)}

En el estudio se utilizó el instrumento Escala de Motivación Académica (EMA). El mismo consiste en un inventario de 28 ítems que contiene razones por las cuales se asiste a clases. Este instrumento fue diseñado con la pregunta: ¿Por qué va a la universidad? así como distintos ítems con las razones para involucrarse en actividades académicas. La respuesta valora el grado de correspondencia de las razones con la percepción individual sobre una escala de siete puntos en escala de Likert.

Las variables definidas en la EMA son la MI (Motivación Intrínseca) y la ME (Motivación Extrínseca). La MI se divide de la siguiente manera: MI para conocer (MIC), MI para rendir o realizar cosas (MIRRCO) y MI para experimentar estimulación (MIPEE). En cuanto a la ME, esta se subdivide en ME de regulación externa (MERE), ME de regulación interna (MERI), ME de identificación (MEI) y amotivación (AMO) (Manassero \& Vázquez, 2000). Asimismo se ha definido una variable denominada total que es la suma de la puntuaciones de $\mathrm{Ml}$ y ME.

Como dicha escala estaba originalmente planteada para ser aplicada a estudiantes universitarios se contextualizó para poder ser utilizada en estudiantes de enseñanza secundaria. La adaptación realizada consistió en sustituir el término universidad por colegio. Posteriormente esta fue sometida a la validación de un grupo de expertos conformado por especialistas en psicología, investigadores estadísticos y de lengua española. Una vez realizado lo anterior se procedió a una validación estadística por medio de la cual se calcularon los alfas de Cronbach en todas las dimensiones. En este procedimiento participaron 60 personas de entre 14 y 16 años de edad.

Luego del análisis se comprobó que la pregunta 1 no correlacionaba (disminuyó el coeficiente r) y se repetía el tópico con las otras preguntas de la misma dimensión, razón por la cual fue eliminada (tabla 2).

Asimismo se diseñó un instrumento de observación con las categorías del estudio (MI, ME y AMO), el que fue utilizado para recopilar aquellos comportamientos y signos que podían ubicarse en las categorías definidas para este estudio. Esta técnica es apropiada para los estudios fenomenológicos (Hernández, Fernández y Baptista, 2010).

Se diseñaron instrumentos para los grupos focales, los que fueron previamente validados por los especialistas.

\section{Análisis de datos}

El estudio se realizó con un enfoque mixto, fenomenológico y cuasiexperimental llamado diseño de grupo control no equivalente (Campbell y Stanley, 2001). Desde la fenomenología, los hallazgos fueron validados mediante la verdad y la validez intersubjetivas a través de las percepciones individuales de los participantes del estudio. Según Sandín (2003, p. 198) la verdad de los participantes es válida en sí misma al formar parte de su propia realidad. A efectos de este estudio, la teoría aplicada sobre motivación es la mencionada por Manassero y Vázquez (2000) como variable dependiente y también tendrá la propiedad de categoría en el análisis cualitativo. Según Manassero y Vázquez esta variable/categoría se subdivide en tres dimensiones -MI, ME y AMO-, también variables dependientes y subcategorías de análisis. A excepción de la AMO, cada una de esas dimensiones se divide en otras subdimensiones que serán estudiadas en el análisis cuantitativo pero no en el cualitativo. 
Las variables independientes de esta investigación son: tiempo (pretest y postest), grupos (control y de estudio) y género (hombre y mujer).

En el análisis cuantitativo se calculó el alfa de Cronbach en todas las escalas, para proceder con la validación del instrumento en el contexto de la población del estudio en Costa Rica. En este punto no se hicieron adecuaciones ya que los especialistas no lo consideraron necesario (ver tabla 2). Una vez finalizada la implementación se realizó un análisis de variancia ANOVA factorial $(2 \times 2 \times 2)$, para el tiempo, el género y los grupos. Todo lo anterior se analizó por medio del S.P.S.S. versión 20.0 para Windows.

Por último se realizó una triangulación múltiple para lograr una combinación de ambos enfoques con el fin de complementar y verificar los hallazgos encontrados mediante la teoría y los diferentes métodos de recolección de datos (Denzin, 2000).

\section{Resultados y análisis}

A continuación se detallarán y explicarán los resultados de la siguiente manera:

Tabla 2. Distribución de los participantes en el estudio, por sexo y por grupo.

\begin{tabular}{cccc} 
& \multicolumn{3}{c}{ Grupo } \\
Sexo & Control & Experimental & Total \\
Hombre & 4 & 13 & 17 \\
Mujer & 11 & 12 & 23 \\
Total & 15 & 25 & 40 \\
\hline
\end{tabular}

La tabla anterior indica la distribución de la muestra. Al finalizar la implementación se constató abandono escolar en ambos grupos: 2 participantes del grupo experimental ( 2 mujeres) y 18 del grupo control (8 hombres y 10 mujeres). Asimismo es importante aclarar que debido a la alta deserción en el grupo control se decidió contactar a desertores para investigar las razones ya que esto podría ser un indicador de AMO.

Respecto a la validación del instrumento de motivación mediante el cálculo del alfa de Cronbach se obtuvieron los siguientes datos:

Tabla 3. Resultados del Alfa de Cronbach de la validación del instrumento de motivación adaptado para secundaria, con un total de 40 estudiantes.

\begin{tabular}{|l|l|}
\hline Escala & Valor alfa \\
\hline $\begin{array}{l}\text { Motivación intrínseca (MI), que comprende Motivación intrínseca para conocer } \\
\text { (Preguntas 2, 9, } 16 \text { y 23) }\end{array}$ & 0,612 \\
\hline Motivación intrínseca para rendir o realizar cosas (Preguntas 6, 13, 20 y 27) & 0,792 \\
\hline Motivación intrínseca para experimentar estimulación (Preguntas 4, 11, 18 y 25) & 0,760 \\
\hline Motivación extrínseca (ME), que engloba regulación externa (Preguntas 1*, 8, 15 y 22) & 0,736 \\
\hline Motivación extrínseca de regulación interna (introyección) (Preguntas 7, 14, 21 y 28) & 0,810 \\
\hline Motivación extrínseca de identificación (Preguntas 3, 10, 17 y 24) & 0,750 \\
\hline Amotivación (AMO) (Preguntas 5, 12,19 y 26) & 0,820 \\
\hline
\end{tabular}

Nota: * esta pregunta fue eliminada para esta adaptación ya que fue la que puntuó muy bajo en el coeficiente. 
De acuerdo con la tabla 3 pueden observarse los resultados de los alfas de Cronbach para todas las escalas. De los mismos solo el coeficiente de la escala MI no tiene el valor mínimo de 0,70. Sin embargo los demás coeficientes tienen un valor aceptable, tal como lo explica Cronbach (1951). Por lo general se anteponen coeficientes de 0,8 a 0,9 pero si no hay otro instrumento se pueden aceptar valores inferiores a estos.

Tabla 4. Dimensiones de la motivación interna.

\begin{tabular}{lcccccccc}
\hline & \multicolumn{3}{c}{ MIC } & \multicolumn{2}{c}{ MIRRCO } & \multicolumn{2}{c}{ MIPEE } & \multicolumn{2}{c}{ MIT } \\
\hline Medición & $F$ & Sig. & $F$ & Sig. & F & Sig. & F & Sig. \\
Tiempo & 0,152 & 0,700 & 0,141 & 0,710 & 6,504 & 0,16 & 0,946 & 0,338 \\
Sexo & 0,681 & 0,415 & 0,213 & 0,648 & 1,906 & 0,177 & 2,033 & 0,164 \\
Grupos & 3,790 & 0,060 & 0,014 & 0,906 & 3,576 & 0,068 & 1,963 & 0,171 \\
Tiempo* sexo & 1,56 & 0,220 & 0,001 & 0,970 & 1,534 & 0,224 & 1,095 & 0,303 \\
Tiempo * grupo & 1,86 & 0,820 & 4,245 & 0,048 & 0,674 & 0,418 & 2,686 & 0,111 \\
Tiempo * sexo & 2,48 & 0,125 & 0,404 & 0,529 & 0,064 & 0,803 & 0,548 & 0,465 \\
* grupo & & & & & & & & \\
Sexo * grupo & 0,540 & 0,468 & 0,167 & 0,686 & 0,806 & 0,376 & 0,545 & 0,466 \\
\hline
\end{tabular}

La tabla 4 muestra que entre los grupos no hubo diferencias estadísticamente significativas en ninguna de las variables comparadas en las diferentes dimensiones de la motivación intrínseca. Sin embargo, en las entrevistas realizadas al estudiantado fue posible percibir una mejora en la motivación debido a la aplicación del AC ya que se observó que en el momento de realizar las actividades hubo una satisfacción experimentada, tal como mencionan los participantes del grupo de estudio: "Si no tuviera nota, vendría como un taller solo a aprender", "A pesar de no gustarnos dibujar disfrutamos mucho de pintar y el poder tomar ciertas decisiones".

Lo anterior concuerda con Orozco (2013) y Manassero y Vázquez (2000) que plantean que el nivel de MI (MIRRCO específicamente) determina la realización de una actividad por la satisfacción experimentada.

Por su parte, lo expresado por Martí y Valls (2006) estaría apoyando dicha postura, la que indica que cuando se involucra directamente al estudiantado en el trabajo por realizar, aumenta su motivación: "Nos gustó mucho trabajar en grupos y salir de la clase. Ojalá todas las clases fueran así, más con el calor que hace aquí" (informante del grupo experimental).

Por último, una de las posibles causas de no encontrar diferencias en los resultados cuantitativos puede deberse a que el instrumento no fue sensible a los cambios en el constructo medido. 
Tabla 5. Dimensiones de la motivación extrínseca.

\begin{tabular}{lcccccccc}
\hline & \multicolumn{2}{c}{ MERE } & \multicolumn{2}{c}{ MERI } & \multicolumn{2}{c}{ MEI } & \multicolumn{2}{c}{ MET } \\
Medición & $F$ & Sig. & $F$ & Sig. & $F$ & Sig. & $F$ & Sig. \\
Tiempo & 0,073 & 0,789 & 0,242 & 0,626 & 0,628 & 0,434 & 0,053 & 0,819 \\
Sexo & 0,013 & 0,912 & 0,211 & 0,649 & 0,057 & 0,813 & 0,000 & 0,988 \\
Grupos & 2,489 & 0,125 & 0,433 & 0,515 & 5,5 & 0,025 & 3,270 & 0,080 \\
Tiempo * sexo & 5,194 & 0,029 & 1,054 & 0,312 & 0,271 & 0,607 & 2,260 & 0,142 \\
$\begin{array}{l}\text { Tiempo * } \\
\text { grupo }\end{array}$ & $0,5,75$ & 0,022 & 3,430 & 0,073 & 5,25 & 0,029 & 7,094 & 0,012 \\
$\begin{array}{l}\text { Tiempo * sexo } \\
\text { * grupo }\end{array}$ & 0,230 & 0,882 & 0,552 & 0,463 & 0,012 & 0,912 & 0,034 & 0,860 \\
Sexo * grupo & 0,268 & 0,608 & 0,053 & 0,819 & 0,829 & 0,369 & 0,247 & 0,622 \\
\hline
\end{tabular}

En la tabla 5 puede observarse que hay diferencias en la variable MEI por grupo (F: 5,50; $\mathrm{p}$ =,025), así como en la interacción tiempo (mediciones) * grupo (F: 5,25; p = 0,29). Esto es, en cuanto a la variable grupo, el grupo de estudio desde el inicio tuvo puntajes mayores de $\mathrm{MEI}$ que el grupo control y así sucedió en la segunda medición. Empero, tal como puede observarse, esto no es un efecto de la implementación de la metodología ya que no hubo diferencias en las variables y en la interacción. Lo mismo ocurrió en la variable MET por grupo (F: 3,27; $p=0,08$ ). Asimismo se observan interacciones significativas en la variable MERE (tiempo * sexo, F: 5,194; $p=0.029$ ) y tiempo * grupo (F: 5,75; $p=$ $0,022)$. No obstante, al no haber diferencias en las variables principales, las interacciones significativas no son representativas de algún hallazgo importante.

Tabla 6. Resultado de las pruebas F y significancia para las variables Mediciones, Sexo, Grupo e Interacciones en la variable AMO Dimensiones de la amotivación.

\begin{tabular}{lcccc}
\hline & \multicolumn{2}{c}{ AMO } & \multicolumn{2}{c}{ MT } \\
Medición & F & Sig. & F & Sig. \\
Tiempo & 0,019 & 0,670 & 0,794 & 0,380 \\
Sexo & 1,640 & 0,210 & 0,090 & 0,770 \\
Grupos & 0,000 & 0,980 & 2,560 & 0,113 \\
Tiempo* sexo & 0,480 & 0,500 & 2,940 & 0,096 \\
Tiempo * grupo & 3,480 & 0,070 & 4,750 & 0,037 \\
Tiempo * sexo * grupo & 0,440 & 0,510 & 0,035 & 0,850 \\
Sexo * grupo & 0,530 & 0,470 & 0,554 & 0,462 \\
\hline
\end{tabular}

En la tabla 6 puede observarse que no hay diferencias en las variables $(p>0,05)$ y solo interacciones que resultan irrelevantes al no existir diferencias en las variables de manera individual. 
En consonancia con lo afirmado por Barnett et al. (2003), de acuerdo con los extractos principales de las observaciones realizadas por los investigadores, al principio de la investigación el grupo control se encontraba muy motivado y, por el contrario, el grupo experimental se encontraba con poca disposición hacia la tarea. Al pasar las semanas se demuestra que, con la intervención del AC el grupo experimental fue incrementando intrínseca y extrínsecamente la motivación y el interés por las tareas realizadas. Esto coincide con los estudios de Tobar (2009) quien señala que el docente debe emplear actividades dinámicas así como conocer las capacidades y limitaciones del estudiantado, lo cual puede ser facilitado con el AC.

Todo lo contrario ocurrió con el grupo control pues al emplear la instrucción tradicional y no hacer uso de las técnicas del AC se observó AMO. Esto evidencia que en el grupo control hubo una percepción de ausencia de motivación tal como expresa un participante al final del estudio: “Eso no es para mí. Tenemos otros intereses más como las académicas y sentimos que materias como música, plásticas o industriales le quitan el tiempo a uno, que se podría aprovechar más con otras materias".

A su vez, uno de los estudiantes desertores comentó lo siguiente: “Mi mamá me sacó porque faltaba mucho a clases. La verdad solo venía a estar en el pasillo o en el Internet, también jugaba mucho futbolín".

Desde el acercamiento cualitativo en el grupo experimental se puede analizar cómo al final de la implementación del AC este funge como elemento propiciador de ME y MI. Tal como indica Chumba (2009), la metodología empleada en las aulas puede ser un factor de deserción escolar, o a la inversa, cuando una metodología como el AC provee herramientas para que el estudiantado mejore el rendimiento académico y se integre a gusto en el centro educativo (p. 8). Este un argumento que respalda lo anterior: "Nos gustó mucho trabajar en grupos y salir de la clase. Ojalá todas las clases fueran así más con el calor que hace aquí". "Iríamos aunque no tuviera nota solo a aprender como si fuera un taller, solo dibujaría lo que me interesa como el dibujo manga" (informante del grupo experimental).

Como análisis final, aunque no hubo diferencias estadísticamente significativas en las variables, se puede constatar que en las percepciones del estudiantado sí hubo una percepción positiva en la $\mathrm{M}$ del grupo experimental en contraste con las del grupo control. Esto se evidencia en lo expresado por los participantes del grupo de estudio: "Disfrutamos mucho de la experiencia, en especial porque se tomaron en cuenta nuestras habilidades, ya que nos gusta más dirigir al grupo, somos más como líderes". Lo anterior coincide con las observaciones realizadas, y que se destaca en la siguiente anotación: "El grupo estudio reacciona de manera increíble, se sienten a gusto trabajando en grupo". Esto va en consonancia con las investigaciones de Pujolàs (2003, p. 8) y García y Dómenech (1997) quienes coinciden en la libertad controlada que debe tener el alumnado para pensar en posibles soluciones, arriesgarse a cometer errores y tomar las decisiones más adecuadas para resolver la tarea con la ayuda del docente que debe saber cómo ganarse la confianza del alumnado.

Por su parte Barnett et al. (2003) hablan sobre la capacidad que debe tener el docente para que, con una actitud humana y comprensiva y sin imponer su criterio, pueda adaptarse al alumnado para que este alcance libertad de expresión con actividades dinámicas (Tobar, 2009). Lo anterior, además de reforzar la relación profesorado-alumnado, contribuye a aumentar la M. Esto se evidencia en el accionar de la profesora en el desarrollo del 
estudio ya que el cambio derivado de la implementación de la metodología permitió que los discentes tomaran mejores decisiones sobre ciertas elecciones sin que mediara una imposición de criterio por parte de la docente. Esto se evidencia en la siguiente nota: "A la hora de escoger los diseños el grupo experimental eligió el diseño del compañero que mejor lo hizo y hubo una satisfacción grupal cuando el resto del grupo elogió el trabajo". Por tanto, y en concordancia con lo afirmado por Martí y Valls (2006), se demuestra que el nivel de $\mathrm{M}$ se incrementa al ser los discentes los que se involucran al $100 \%$ en la tarea.

De acuerdo con González Lutz, M.I. (comunicación personal del 26 de mayo de 2014) la $M$ no se da en un momento sino que es un proceso que se refleja en la personalidad del individuo. Al analizar los datos puede observarse que el instrumento de motivación no fue sensible a los cambios en la motivación del estudiantado. Esto es notorio por la evidencia que proyectaron los datos en la investigación cualitativa, como por ejemplo, el hecho de que el grupo control presentó una deserción de 18 personas y el grupo experimental solo de 2. Este hecho evidencia que el grupo experimental tuvo mayores niveles de motivación positiva que el grupo control. En concordancia con lo indicado por Manassero y Vázquez (2000), estos estudiantes tuvieron más motivación en específico como una acción de éxito o fracaso, situación que se ve reflejada en el siguiente comentario de un informante del grupo experimental: "Tratábamos de superarlo y continuábamos con el trabajo. Al principio nos asustamos cuando algo no nos salía bien, pero nos organizábamos rápido y superábamos el reto".

Lo anterior concuerda con lo indicado por Johnson y Johnson (1991) quienes exponen que las metodologías emergentes y humanistas como la del AC versus metodologías academicistas hacen que las personas -específicamente el estudiantado- tiendan más a querer estudiar, ya que dichas metodologías propician la participación de los jóvenes como entes protagonistas en el proceso de enseñanza-aprendizaje. No así la represión. Esto va en contraposición con otro tipo de metodologías centradas en el profesor en las que, tal como indican Mas y Medinas (2007), el estudiantado se ve anulado y por ende tiende a reprimir la motivación hacia las clases. Eso podría desembocar en deserción. Esto concuerda con Díaz-Barriga y Hernández (2004) quienes afirman que el docente debe actuar como guía y ofrecer la dirección pertinente al estudiante en cada situación de aprendizaje, así como con Liao (2006), quien reafirma que el AC incide en la motivación debido a que propicia resultados de eficacia, desenvolvimiento y alto rendimiento. Esto se evidencia en el siguiente comentario del informante del grupo experimental: “...es como un descanso entre tanto corre corre de otras materias".

Por último se resaltan dos aspectos a nivel cualitativo observados durante la ejecución de la metodología aplicada. Los mismos confirman que el objetivo del proyecto fue alcanzado.

Primer aspecto: el número de deserciones en el grupo control (con metodología tradicional):

"Yo solo vengo al cole porque me obliga mi mamá". "Al no asistir a lecciones mi mamá tomó la decisión de sacarme del colegio, porque ayudaba más en la casa que vagueando en el colegio" (Desertor 1).

"Tenía muchas ganas de seguir estudiando, me gustaban mucho las plásticas pero me costaban mucho las materias académicas y al llevar por segundo año consecutivo rojo las materias académicas mi familia tomó la decisión de sacarme de estudiar y ponerme a cuidar a mis hermanos menores" (Desertor 2). 
Otro aspecto a destacar es que en el grupo experimental la deserción fue menor, evidenciando que, a pesar de la falta de sensibilidad del instrumento con respecto al tiempo de implementación debido a que solo se hizo una medición, el AC tuvo un efecto positivo en el grupo experimental en cuanto a la permanencia. Por tanto, se concluye que el AC promueve la M.

\section{Referencias bibliográficas}

Barnett, L. et al. (2003). Motivación, tratamiento de la diversidad y rendimiento académico. El aprendizaje cooperativo. Venezuela: Editorial Laboratorio Educativo.

Campbell, D. y Stanley, J. (2001). Diseños experimentales y cuasiexperimentales en la investigación social. Argentina: Amorrortu Editores.

Chenich, G. y Santos, G. (2005). Propuesta de aprendizaje basado en proyecto y trabajo colaborativo: experiencia de un curso en línea. REDIE. Revista Electrónica de Investigación Educativa, 7(2). https://www.redalyc.org/articulo.oa?id=15507204

Chumba, R. (2009). El aprendizaje cooperativo y la deserción escolar en la licenciatura en contaduría y administración del Centro de Estudios Superiores CTM. (Tesis de maestría, Universidad Autónoma de Yucatán). https://docplayer.es/5687911-El-aprendizajecooperativo-y-la-desercion-escolar-en-la-licenciatura-en-contaduria-y-administraciondel-centro-de-estudios-superiores-ctm.html

Cokley, K., Bernard, N., Cunningham, D., \& Motoike, J. (2001). A Psychometric Investigation of the Academic Motivation Scale Using a United States Sample. Measurement and Evaluation in Counseling and Development, 34(2), 109-119. https://doi.org/10.1080/07481 756.2001.12069027,

https://www.researchgate.net/publication/232466311_A_psychometric_investigation_of the_Academic_Motivation_Scale_using_a_United_States_sample

Cronbach, L. (1951). Coefficient alpha and the internal structure of test. Psycometrika, 311(3), 297-334). https://doi.org/10.1007/BF02310555. https://link.springer.com/ article/10.1007/BF02310555

Denzin, N. (2000). What is Triangulation? http://jthomasniu.org/class/Handouts/triangdenzin

Díaz-Barriga, F. y Hernández, G. (2004). Estrategias docentes para un aprendizaje significativo (2. ${ }^{a}$ ed.). México: Mc Graw Hill. http://formacion.sigeyucatan.gob.mx/formacion/ materiales/4/4/ d2/p1/5.\%20estrategias_docentes_para_un_aprendizaje_significativo.pdf

Duran, D. y Vidal, V. (2004). Tutoría entre iguales: de la teoría a la práctica. España: Editorial Graó.

Ferreiro, R. y Espino, M. (2009). El ABC del aprendizaje cooperativo: Trabajo en equipo para aprender y enseñar. México: Editorial Trillas. http://www.habilidadesparaadolescentes. com/archivos/El_Abc_del_aprendizaje_cooperativo.pdf

García, F. y Dómenech, F. (1997). Motivación, aprendizaje y rendimiento escolar. R. E. M. E. Revista Electrónica de Motivación y Emoción, 1(0). http://reme.uji.es/articulos/pa0001/texto. html 
Gilbert, I. (2005). Motivar para aprender en el aula. Las siete claves de la motivación escolar. Barcelona: Editorial Paidós. http://www.xtec.cat/ ilopez15/materials/ambitpedagogic/ motivacio/motivarparaaprenderenelaula.pdf

Hauser, A. (1978). Historia social de la literatura y el arte. Barcelona: Editorial Calabria. https://es.scribd.com/doc/34401771/Hauser-Arnold-Historia-Social-de-La-Literatura-YEl-Arte-Tomo-1

Hernández, R., Fernández, C. y Baptista, P. (2010). Metodología de la investigación (5. a ed.). México: Editorial McGraw Hill. https://www.esup.edu.pe/descargas/dep_investigacion/ Metodologia\%20de\%20la\%20investigaci\%C3\%B3n\%205ta\%20Edici\%C3\%B3n.pdf

Johnson, D. y Johnson, R. (1999). Aprender juntos y solos. Buenos Aires: Grupo Editorial Aique S.A.

Johnson, D., Johnson, R. \& Holubec, E. (1994). The new circles of learning: Cooperation in the classroom and school. Alexandria, VA: Association for Supervision and Curriculum Development.

Johnson, D., Johnson, R. y Holubec, E. (1994). El aprendizaje cooperativo. Buenos Aires: Editorial Paidós SAICF.

Johnson, D., Johnson, R. \& Smith, K. (1998). Active learning: Cooperation in the college classroom. The Annual Report of Educational Psychology in Japan (47). https://www. researchgate.net/publication/234568124_Active_Learning_Cooperation_in_the_College_ Classroom/citation/download

Lara, S. (2001). Una estrategia eficaz para fomentar la cooperación. Estudios Sobre Educación, 1, 99-110. https://www.unav.edu/publicaciones/revistas/index.php/estudiossobre-educacion/article/view/25688

Liao, H.-C. (2006). Effects of Cooperative Learning on Motivation, Learning Strategy Utilization, and Grammar Achievement of English Language Learners in Taiwan (Tesis doctoral). University of New Orleans, Estados Unidos. https://scholarworks.uno.edu/ $\underline{\mathrm{td} / 329}$

López, M. (2009). Efectos del aprendizaje cooperativo en las habilidades sociales, la educación intercultural y la violencia escolar: un estudio bibliométrico de 1997 a 2007 (Tesis doctoral, Universidad de Alicante). http://www.cervantesvirtual.com/obra/efectosdel-aprendizaje-cooperativo-en-las-habilidades-sociales-la-educacion-intercultural-y-laviolencia-escolar-un-estudio-bibliometrico-de-1997-a-2007--0/

Manassero, M. y Vázquez, A. (2000). Análisis empírico de dos escalas de motivación escolar. Revista electrónica de motivación y emoción, 3(5-6). http://reme.uji.es/articulos/ amanam5171812100/texto.html

Mas, C. y Medinas, M. (2007). Motivaciones para el estudio en universitarios. Anales de psicología, 23(1), (17-24). https://www.um.es/analesps/v23/v23_1/03-23_1.pdf

Martí, J. y Valls, M. (2009). Motivación y juego en el aula: la implicación del alumno en las clases de E/LE. Foro de profesores de E/LE, 5 (1-10). https://dialnet.unirioja.es/servlet/ articulo?codigo $=4892272$ 
Ministerio de Educación Pública de Costa Rica. (2009). Proyecto de ética, estética y ciudadanía. Programas de estudio. Artes plásticas. Tercer ciclo de educación general básica y educación diversificada. Costa Rica: Ministerio de Educación Pública. https://www.mep.go.cr/sites/ default/files/descargas/programas-de-estudio/artesplasticas3cicloydiversificada.pdf

Duran, D. y Monereo, C. (2012). Entramado: Métodos de aprendizaje cooperativo y colaborativo. Barcelona: Horsori.

Muñoz, J. M. (2009). Cuatro recursos didácticos frente a la falta de motivación en las aulas. Revista Digital Innovación y experiencias educativas, 45 (6. ${ }^{a}$ ed.). https://archivos.csif.es/ archivos/andalucia/ensenanza/revistas/csicsif/revista/pdf/Numero_15/JOSE\%20MARIA_ MUNOZ_2.pdf

Orozco, R. (2013). Motivación profesional, estilos de aprendizaje y satisfacción del estudiante en el contexto del aula de educación física. (Tesis doctoral, Universidad estatal a distancia). http://repositorio.uned.ac.cr/reuned/handle/120809/1005

Panitz, T. (1999). Benefits of Cooperative Learning in Relation to Student Motivation. In M. Theall (Ed.), Motivation from within: Approaches for Encouraging Faculty and Students to Excel, New Directions for Teaching and Learning (pp. 59-68). San Francisco, CA: Josey-Bass Publishing.

Pujolàs, P. (2003). El aprendizaje cooperativo: algunas ideas prácticas. http://www.deciencias. net/convivir/1.documentacion/D.cooperativo/AC_Algunasideaspracticas_Pujolas_21p.pdf

Romo, M. (1997). Psicología de la creatividad. Barcelona: Editorial Paidós.

Ruiz, D. (2012). La influencia del trabajo cooperativo en el aprendizaje del área de economía en la enseñanza secundaria (Tesis doctoral, Universidad de Valladolid, Facultad de Educación y Trabajo Social). http://uvadoc.uva.es/handle/10324/2729

Sandín, M. (2003). Investigación cualitativa en educación. Fundamentos y tradiciones. Madrid: McGraw-Hill.

Tobar, M. (2009). Técnicas de motivación para las artes plásticas. https://docplayer. es/6208547-Tecnicas-de-motivacion-para-las-artes-plasticas.html

\section{(cc) BY}

Este artículo está sujeto a una licencia internacional Creative Commons Attribution 4.0.

*,***y**** Ministerio de Educación y Cultura, Costa Rica.

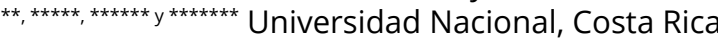

${ }^{1}$ Licenciado en Pedagogía con énfasis en Didáctica, Universidad Nacional (Costa Rica). Profesor de Artes Plásticas, Ministerio de Educación Pública (Costa Rica).

2Profesora de investigación, división de Educología; Asesora, revistas científicas, repositorios, acceso abierto y ciencia abierta, Vicerrectoría de Investigación, Universidad Nacional (Costa Rica). Investigadora en ciencia abierta y pedagogía.

${ }^{3}$ Profesora de Artes Plásticas, Ministerio de Educación Pública (Costa Rica). 
${ }^{4}$ Licenciada en Pedagogía con énfasis en Didáctica, Universidad Nacional (Costa Rica). Profesora de Artes Plásticas, Ministerio de Educación Pública (Costa Rica).

${ }^{5}$ Máster en Estadística, Licenciado en Enseñanza de la Matemática, Universidad Nacional (Costa Rica). Profesor e investigador, Maestría en Economía del Desarrollo, Escuela de Estadística y Escuela de Matemáticas, Universidad (Costa Rica).

${ }^{6}$ Investigadora, Escuela de Economía, Universidad Nacional (Costa Rica). Editora adjunta, revista Economía y Sociedad, Costa Rica.

${ }^{7}$ Máster en Planificación Curricular, Licenciada en Ciencias de la Educación con énfasis en Didáctica del Español y Profesora e investigadora, división de Educología, Universidad Nacional (Costa Rica). Investigadora en formación docente, lectura, escritura y currículum.

\section{Notas}

Aprobación final del artículo, editora responsable Mag. Verónica Zorrilla de San Martín

Contribución de autoría: La concepción del trabajo científico pertenece a Efraín Romero Artavia, Andrea Mora Campos, Wendy González Víquez, Patricia Renee Garbanzo Hernández, José Andrey Zamora-Araya y Silvia García Vargas. La recolección, interpretación y análisis de datos estuvo a cargo de Efraín Romero Artavia, Andrea Mora Campos, Wendy González Víquez, Patricia Renee Garbanzo Hernández, José Andrey Zamora-Araya y Fabiola Quirós Segura.

La redacción y revisión del manuscrito fue realizada por Efraín Romero Artavia, Andrea Mora Campos, José Andrey Zamora-Araya, Fabiola Quirós Segura, Silvia García Vargas. Todos los autores revisaron y aprobaron el contenido final.

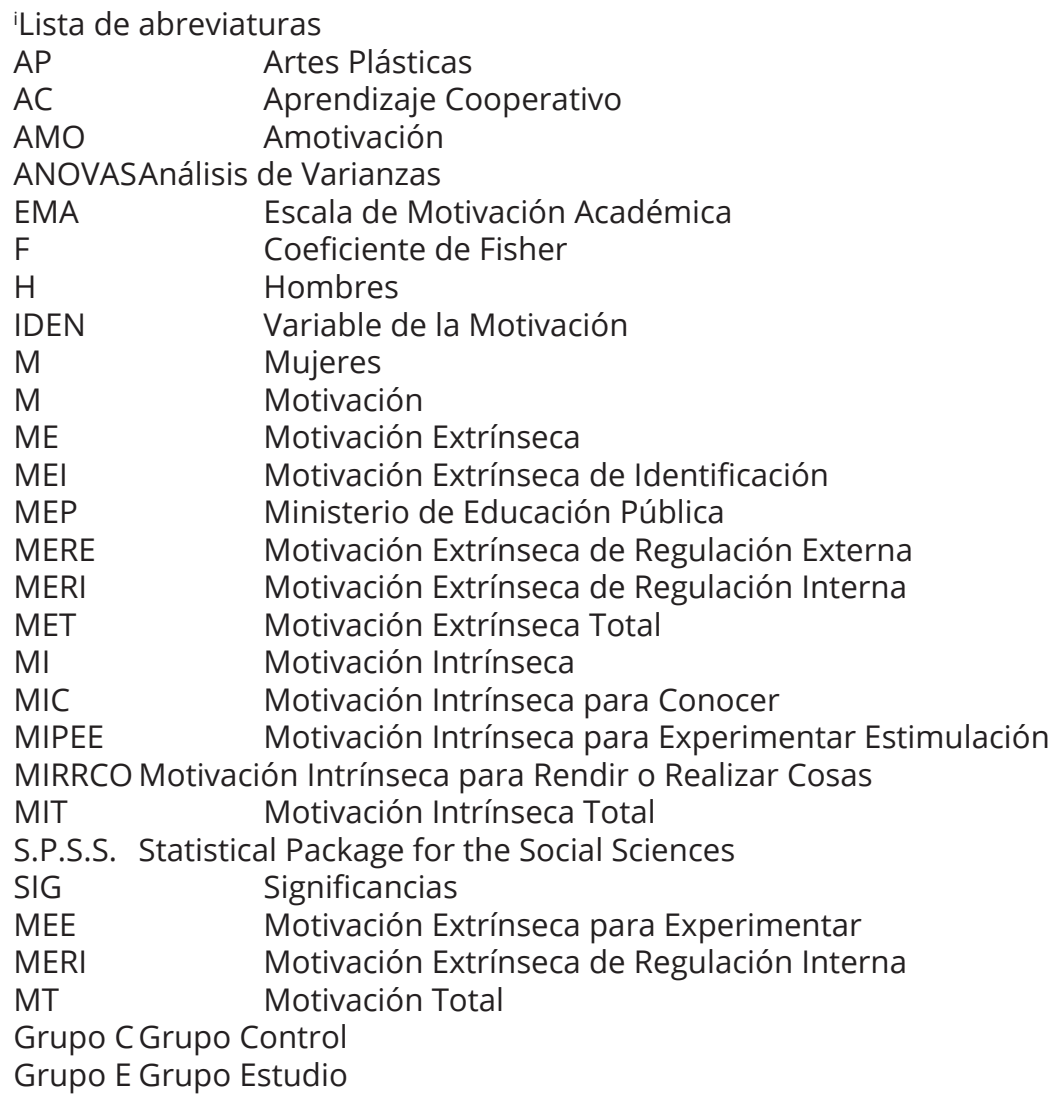

